Tohoku J. Exp. Med., 2007, 212, 91-99

\title{
A Palatinose-Based Balanced Formula Improves Glucose Tolerance, Serum Free Fatty Acid Levels and Body Fat Composition
}

\author{
Toshinide Oizumi, ${ }^{1}$ Makoto Daimon,,${ }^{1}$ Yumi Jimbu, ${ }^{1}$ Wataru Kameda, ${ }^{1}$ \\ Nobuko Arawaka, ${ }^{1}$ Hiroshi Yamaguchi, ${ }^{1}$ Hiroshi Ohnuma, ${ }^{1}$ Hajime Sasaki ${ }^{2}$ and \\ TAKEO KATO ${ }^{1}$ \\ ${ }^{1}$ Department of Neurology, Hematology, Metabolism, Endocrinology and Diabetology \\ (DNHMED), Yamagata University School of Medicine, Yamagata, Japan \\ ${ }^{2}$ Food Science Institute, Meiji Dairies Corporation, Odawara, Japan
}

Oizumi, T., Daimon, M., Jimbu, Y., Kameda, W., Arawaka, N., Yamaguchi, H., Ohnuma, H., Sasaki, H. and Kato, T. A Palatinose-Based Balanced Formula Improves Glucose Tolerance, Serum Free Fatty Acid Levels and Body Fat Composition. Tohoku J. Exp. Med., 2007, 212 (2), 91-99 — Palatinose is a disaccharide present in honey, which has the characteristics of delayed digestion and absorption. We developed a palatinosebased balanced formula (PBF) and reported its beneficial effects on metabolic syndromerelated parameters in rats. To examine the effects of PBF in humans, we here conducted a crossover study using twenty-three subjects with impaired glucose tolerance. The subjects were divided into two groups: intervention to control (I/C) and control to intervention $(\mathrm{C} / \mathrm{I})$ groups. The I/C group consumed PBF (250 kcal) together with foods that were $250 \mathrm{kcal}$ less than their usual breakfast (intervention meal) for the first 12 weeks, followed by their usual breakfast (control meal) for the last 12 weeks. The protocol for the C/I group was opposite in order: the control meal for the first 12 weeks, followed by the intervention meal for the last 12 weeks. In the first 12-week period, the intervention meal decreased 2-hr plasma glucose levels after 75 -g oral glucose tolerance test $(-15.7 \pm 20.1 \%$ change $)$, while the control meal did not $(0.8 \pm 31.6 \%$ change $)$. The difference between these changes was significant $(p=0.038)$. The similar results were obtained from the comparison of the changes between the first and the last 12-week periods in the two groups combined (intervention vs control: $-11.8 \pm 22.5$ vs $11.2 \pm 30.2 \%$ change, $p=0.024)$. PBF also had the beneficial effects on serum free fatty acids levels and visceral fat area. In conclusion, PBF consumption has beneficial effects on metabolic syndrome-related parameters in humans. — palatinose; free fatty acid; visceral fat area; glucose tolerance; metabolic syndrome

(C) 2007 Tohoku University Medical Press

As shown in many studies, sustained hyperglycemia is a risk factor for both microvascular and macrovascular (or cardiovascular) complica- tions in type 2 diabetes (DM) (Laakso and Lehto 1997; UK Prospective Diabetes Study group 1998; Bretzel et al. 1998), while postprandial

Received March 2, 2007; revision accepted for publication April 13, 2007.

Correspondence: Makoto Daimon, Department of Neurology, Hematology, Metabolism, Endocrinology and

Diabetology (DNHMED), Yamagata University School of Medicine, 2-2-2 Iida-Nishi, Yamagata 990-9585, Japan.

e-mail:mdaimon@med.id.yamagata-u.ac.jp 
hyperglycemia has also been considered a risk factor for cardiovascular complications (Tominaga et al. 1999; Temelkova-Kurktscheive et al. 2000; Risso et al. 2001; Chiasson et al. 2002; Hanefeld et al. 2004; Nakagami et al. 2004). Many experimental and epidemiological studies have shown that increased postprandial plasma glucose (PG) levels may have equally or even more harmful effects than fasting hyperglycemia (Tominaga et al. 1999; Temelkova-Kurktscheive et al. 2000; Risso et al. 2001; Nakagami et al. 2004), and the reduction of postprandial plasma glucose levels delays the development of cardiovascular complications (Chiasson et al. 2002; Hanefeld et al. 2004). Postprandial hyperglycemia is a recognized consequence of the effects of both insulin resistance and the impairment of early insulin secretion in response to an oral glucose load (Reaven and Miller 1968; Bruce et al. 1988; Lillioja et al. 1988). However, inversely, both insulin resistance and the impairment of early insulin secretion in response to an oral glucose load can, at least initially, be somewhat improved by reducing postprandial glucose levels (Savage et al. 1979; Laedtke et al. 2000). Furthermore, in most cases, postprandial hyperglycemia seems to occur for a significant period before the diagnosis of overt DM. Therefore, the early treatment of subjects with postprandial hyperglycemia appears to be important. Although the administration of a $\alpha$-glucosidase inhibitor, acarbose, has been identified as an effective treatment for delaying the development of cardiovascular complications in subjects with postprandial hyperglycemia or impaired glucose tolerance (IGT) (Chiasson et al. 2002; Hanefeld et al. 2004), lifestyle intervention is as effective as or even more effective than drug intervention (Eriksson et al. 1998; Oldroyd et al. 2001; Uusitupa et al. 2003). However, it is difficult for ordinary subjects to persevere with lifestyle intervention, and indeed the cost was even higher than the cost for drug intervention even if when it was effective (Hernan et al. 2003).

Palatinose (isomaltulose) is a disaccharide present in honey, which has shown promise as a noncariogenic caloric sweetener (Siddiqui et al. 1967; Lingström et al. 1997). Although palati- nose is completely absorbed, it has the specific characteristics of delayed digestion and absorption (Dahlquist et al. 1963; Lina et al. 2002). We have developed a novel palatinose-based enteral formula, designated PBF, as a possible therapeutic means for improving postprandial hyperglycemia, and thus its accompanying conditions and complications (Arai et al. 2004). As shown previously, PBF suppressed postprandial hyperglycemia after bolus intragastric ingestion, reduced visceral fat accumulation and serum triglyceride levels, and improved insulin sensitivity in rats (Arai et al. 2004).

In this report, we have extended our study on the effects of PBF to humans. Furthermore, we have designed a study protocol with which it is easy for subjects to persevere and less expensive than using other drugs or intervention means, which can be applied in ordinary clinical practice; we did not ask the subjects to change their lifestyle during the study period (12 weeks) apart for consuming $250 \mathrm{ml}$ of PBF per day at breakfast. We found that PBF was effective in humans even under this condition.

\section{SubJects ANd Methods}

\section{Subjects}

Twenty-four (F/M: 10/14) subjects were recruited from the residents of a town in Yamagata prefecture, which is located about $400 \mathrm{~km}$ north of Tokyo, Japan, and who had been identified as having IGT at health care examinations held one year before this study. This study was approved by the Ethics Committee of Yamagata University School of Medicine, and informed consent to participate in this study was obtained from the participants.

\section{Study protocol and traits examined}

A crossover design (two 12-week intervention periods) was used to measure treatment effects. The subjects were randomized into two groups for sex and age (intervention to control $[\mathrm{I} / \mathrm{C}]$ and control to intervention $[\mathrm{C} / \mathrm{I}]$ groups). The subjects of the $\mathrm{I} / \mathrm{C}$ group consumed at breakfast a $250 \mathrm{ml}$ can $(250 \mathrm{kcal})$ of PBF (Inslow, previously designated as MHN-01; the composition are reported previously (Arai et al. 2004), as shown in Table 1; Meiji Dairies Corporation, Odawara) together with foods that were $250 \mathrm{kcal}$ less than their usual breakfast to 
TABLE 1. Composition of the palatinose-based balanced formula (PBF).

\begin{tabular}{cl}
\hline $\begin{array}{c}\text { Energy }(\mathrm{kJ} / \mathrm{ml}) \\
(\mathrm{kJ} / \mathrm{g})\end{array}$ & 4.19 \\
Energy balance & 19.4 \\
Protein (energy \%) & \\
Milk protein $(\%)^{*}$ & 20.0 \\
Fat (energy\%) & $(100)$ \\
SFA $(\%)^{* * *}$ & 29.7 \\
MUFA $(\%)^{* *}$ & $(10.9)$ \\
PUFA $(\%)^{* *}$ & $(72.3)$ \\
Other fatty acid $(\%)^{* *}$ & $(15.1)$ \\
Carbohydrate (energy \%) & $(1.7)$ \\
Sucrose $(\%)^{* * *}$ & 50.3 \\
Branched dextrin $(\%)^{* * *}$ & $(0)$ \\
Dextrin $(\%)^{* * *}$ & $(23.9)$ \\
Palatinose $(\%)^{* * *}$ & $(0)$ \\
Xylitol $(\%)^{* * *}$ & $(55.7)$ \\
Other carbohydrate $(\%)^{* * *}$ & $(5.3)$ \\
\hline
\end{tabular}

${ }^{*} \%$ of nutrient in protein; ${ }^{* *} \%$ of nutrient in fat; ${ }^{* * *} \%$ of nutrient in carbohydrate.

maintain their usual total energy intake at breakfast as the intervention in the first 12-week period (period 1), while the $\mathrm{C} / \mathrm{I}$ group subjects consumed their usual breakfast as a Control in period 1. The subjects were advised by medical staff before the study and during the study period how to eat their usual diets to maintain their usual total energy intake in a day. After period 1, the subjects of each group exchanged their protocol for the following 12-week period (period 2). This design allowed the treatment effects to be measured between the study groups (namely between the different subjects) in the same study period, or period 1 , as well as in the same subjects in a crossover comparison of periods 1 and 2 . This study was started in January 2004. Since the number of subjects recruited was 24 , each study group had 12 subjects. However, one subject initially assigned to the $\mathrm{C} / \mathrm{I}$ group did not attend, and one subject from the C/I group insisted on moving to the $\mathrm{I} / \mathrm{C}$ group at the baseline examination. The subjects in the $\mathrm{C} / \mathrm{I}$ and the $\mathrm{I} / \mathrm{C}$ groups were therefore 10 and 13 , respectively. This did not affect the randomization of the subjects for sex and age, as shown in Table 2. Compliance with PBF consumption was recorded by daily self-documentation, and all subjects were found to have consumed PBF at more than $95 \%$ of the scheduled times. All subjects were advised to maintain their usual lifestyle throughout the study periods except for breakfast.

The following characteristics were analyzed at the baseline ( 0 week) and the end (12 [crossover] and 24 weeks) of the each study period: height, body weight, results (PG and insulin) of a $75 \mathrm{~g}$ oral glucose tolerance test (OGTT), HbA1c, waist circumference, hip circumference, waist-to-hip ratio (WHR), body mass index (BMI), percent body fat, abdominal fat composition (area) at the L4-L5 level, an insulin resistance index assessed by homeostasis model assessment (HOMA-IR), systolic and diastolic blood pressures (BPs), total serum cholesterol, serum triglyceride, serum high-density lipoprotein (HDL) cholesterol, serum free fatty acid (FFA), serum remnant-like particle (RLP) cholesterol, and abdominal fat areas. Percent body fat was assessed based on the principles of bioelectrical impedance (Jebb et al. 2000). Abdominal (total and visceral) fat areas (density: -50 to -250 Hounsfield units) were obtained by CT scan in a tomographic slice at the umbilical level as previously reported (Ribeiro-Filho et al. 2003). The baseline characteristics of the study groups are shown in Table 2. Glucose tolerance was diagnosed according to the 1999 World Health Organization criteria (WHO 1999). 
Statistical analysis

Data are given as the means \pm S.D. The statistical significance of differences in the baseline characteristics between the groups was assessed by student's $t$-test. The differences in the characteristic values in each study period in each group were evaluated by student's paired $t$-test. The differences in the percent change in the characteristic values from the baseline to crossover (namely, in period 1) between the groups were evaluated by student's $t$-test as well as Mann Whitney's U-test, and the differences in those from the beginning to the end of each study period between periods 1 and 2 were evaluated by Wilcoxon test. A value of $p<0.05$ was accepted as significant.

\section{RESULTS}

Baseline characteristics of the study groups

Although the subjects were randomized into two groups for sex and age, there were substantial differences in several other characteristics at the baseline between the groups, as shown in Table 2. Hip circumference and abdominal fat areas were

TABLE 2. Baseline characteristics of the study groups.

\begin{tabular}{|c|c|c|c|}
\hline Traits & $\begin{array}{c}\text { Control to } \\
\text { intervention }(\mathrm{C} / \mathrm{I})\end{array}$ & $\begin{array}{l}\text { Intervention to } \\
\text { control (I/C) }\end{array}$ & $p$ \\
\hline Number & 10 & 13 & \\
\hline $\operatorname{Sex}(\mathrm{F} / \mathrm{M})$ & $4 / 6$ & $5 / 8$ & 0.722 \\
\hline Age (yr) & $61.6 \pm 0.5$ & $60.5 \pm 3.5$ & 0.319 \\
\hline Height (cm) & $156.3 \pm 6.6$ & $159.0 \pm 8.3$ & 0.414 \\
\hline Body weight (kg) & $58.3 \pm 9.2$ & $64.4 \pm 7.1$ & 0.088 \\
\hline Waist circumference $(\mathrm{cm})$ & $79.8 \pm 7.7$ & $86.2 \pm 7.6$ & 0.063 \\
\hline Hip circumference $(\mathrm{cm})$ & $89.9 \pm 4.1$ & $95.0 \pm 3.5$ & $0.004^{*}$ \\
\hline Waist-to-hip ratio & $0.887 \pm 0.059$ & $0.906 \pm 0.060$ & 0.454 \\
\hline Body mass index $\left(\mathrm{kg} / \mathrm{m}^{2}\right)$ & $23.8 \pm 2.6$ & $25.6 \pm 3.1$ & 0.154 \\
\hline Percent body fat $(\%)$ & $27.3 \pm 5.9$ & $30.6 \pm 8.0$ & 0.299 \\
\hline Abdominal fat area: Total $\left(\mathrm{cm}^{2}\right)$ & $147.1 \pm 65.9$ & $218.5 \pm 87.7$ & $0.044^{*}$ \\
\hline Visceral $\left(\mathrm{cm}^{2}\right)$ & $56.1 \pm 30.6$ & $83.1 \pm 37.3$ & 0.077 \\
\hline \multicolumn{4}{|l|}{ OGTT: } \\
\hline Fasting plasma glucose (mg/dl) & $103.9 \pm 11.2$ & $101.4 \pm 12.8$ & 0.628 \\
\hline 1-hr plasma glucose (mg/dl) & $200.3 \pm 49.7$ & $200.8 \pm 59.2$ & 0.984 \\
\hline 2-hr plasma glucose (mg/dl) & $155.6 \pm 44.8$ & $164.3 \pm 54.1$ & 0.685 \\
\hline Fasting serum insulin $(\mu \mathrm{U} / \mathrm{ml})$ & $5.3 \pm 2.9$ & $7.5 \pm 3.6$ & 0.135 \\
\hline 1-hr serum insulin $(\mu \mathrm{U} / \mathrm{ml})$ & $47.3 \pm 33.4$ & $37.6 \pm 25.9$ & 0.461 \\
\hline 2-hr serum insulin $(\mu \mathrm{U} / \mathrm{ml})$ & $36.5 \pm 36.8$ & $35.8 \pm 21.5$ & 0.960 \\
\hline $\operatorname{HbA1c}(\%)$ & $5.28 \pm 0.6$ & $5.41 \pm 0.5$ & 0.566 \\
\hline HOMA-IR & $1.36 \pm 0.72$ & $1.84 \pm 0.88$ & 0.180 \\
\hline Systolic blood pressure (mmHg) & $138.2 \pm 13.9$ & $132.5 \pm 16.5$ & 0.387 \\
\hline Diastolic blood pressure $(\mathrm{mmHg})$ & $85.0 \pm 8.8$ & $79.7 \pm 12.9$ & 0.279 \\
\hline Total cholesterol (mg/dl) & $209.6 \pm 44.0$ & $195.1 \pm 35.2$ & 0.389 \\
\hline Triglyceride (mg/dl) & $110.0 \pm 82.7$ & $128.2 \pm 80.4$ & 0.601 \\
\hline HDL cholesterol (mg/dl) & $58.0 \pm 18.8$ & $57.8 \pm 12.2$ & 0.981 \\
\hline Free fatty acid (mEq/l) & $0.750 \pm 0.346$ & $0.911 \pm 0.180$ & 0.163 \\
\hline RLP cholesterol (mg/dl) & $5.80 \pm 5.46$ & $5.71 \pm 2.78$ & 0.958 \\
\hline
\end{tabular}

Values are mean \pm S.D. $p<0.05$ is indicated by * 
significantly higher in the I/C group than in the $\mathrm{C} / \mathrm{I}$ group, indicating that the subjects in the I/C group were more obese in the upper body. Furthermore, several other characteristics such as body weight, waist circumference and abdominal visceral fat area (VFA) tended to be higher in the $\mathrm{I} / \mathrm{C}$ group than in the C/I group. These differences between the groups mentioned above were therefore considered throughout this study.
Changes in the characteristic values in period 1 As shown in Table 3, VFA, 2-hr PG levels and serum FFA levels at the end of period 1 (12 weeks) were significantly lower than at the baseline ( 0 week) in the I/C group, while no characteristic was significantly different between the beginning (baseline) and end of period 1 in the $\mathrm{C} / \mathrm{I}$ group, except for the hip circumference and diastolic BP. We next evaluated the significance

TABLE 3. Change in the characteristic values of the study group in the study periods.

\begin{tabular}{|c|c|c|c|c|c|c|}
\hline \multirow{4}{*}{ Characteristics } & \multicolumn{3}{|c|}{ Control to intervention $(\mathrm{C} / \mathrm{I})$} & \multicolumn{3}{|c|}{ Intervention to control (I/C) } \\
\hline & \multicolumn{3}{|c|}{ Intervention (Period 2) } & \multicolumn{3}{|c|}{ Control (Period 2) } \\
\hline & \multicolumn{2}{|c|}{ Control (Period 1) } & \multirow[b]{2}{*}{24 weeks } & \multicolumn{2}{|c|}{ Intervention (Period 1) } & \multirow[b]{2}{*}{24 weeks } \\
\hline & Baseline & 12 weeks & & Baseline & 12 weeks & \\
\hline Body weight (kg) & $58.3 \pm 9.2$ & $58.3 \pm 9.1$ & $56.5 \pm 8.5^{*}$ & $64.4 \pm 7.1$ & $65.4 \pm 8.1$ & $62.9 \pm 6.7^{*}$ \\
\hline Waist circumference $(\mathrm{cm})$ & $79.8 \pm 7.7$ & $79.9 \pm 9.7$ & $78.2 \pm 8.2$ & $86.2 \pm 7.6$ & $85.7 \pm 6.2$ & $83.9 \pm 8.1$ \\
\hline Hip circumference $(\mathrm{cm})$ & $89.9 \pm 4.1$ & $92.0 \pm 4.2^{*}$ & $89.6 \pm 4.5^{*}$ & $95.0 \pm 3.5$ & $94.8 \pm 3.4$ & $94.1 \pm 4.2$ \\
\hline Waist-to-hip ratio (\%) & $88.7 \pm 5.9$ & $86.8 \pm 8.6$ & $87.2 \pm 6.5$ & $90.6 \pm 6.0$ & $90.4 \pm 5.3$ & $89.1 \pm 6.2$ \\
\hline Body mass index $\left(\mathrm{kg} / \mathrm{m}^{2}\right)$ & $23.8 \pm 2.6$ & $23.7 \pm 2.6$ & $23.0 \pm 2.3^{*}$ & $25.6 \pm 3.1$ & $25.3 \pm 3.0$ & $25.0 \pm 3.2^{*}$ \\
\hline Percent body fat $(\%)$ & $27.3 \pm 5.9$ & $26.6 \pm 6.3$ & $24.1 \pm 5.4^{* *}$ & $30.6 \pm 8.0$ & $30.9 \pm 8.2$ & $28.7 \pm 8.0^{* * *}$ \\
\hline \multirow{2}{*}{$\begin{aligned} \text { Abdominal fat area: } & \text { Total }\left(\mathrm{cm}^{2}\right) \\
& \text { Visceral }\left(\mathrm{cm}^{2}\right)\end{aligned}$} & $147.1 \pm 65.9$ & $154.1 \pm 78.1$ & $117.5 \pm 74.9^{*}$ & $218.5 \pm 87.7$ & $203.0 \pm 92.6$ & $205.8 \pm 87.4$ \\
\hline & $56.1 \pm 30.6$ & $53.6 \pm 39.6$ & $38.6 \pm 23.6$ & $83.1 \pm 37.3$ & $65.5 \pm 31.4^{*}$ & $70.6 \pm 38.6$ \\
\hline \multicolumn{7}{|l|}{ OGTT: } \\
\hline Fasting plasma glucose (mg/dl) & $103.9 \pm 11.2$ & $101.9 \pm 18.6$ & $102.7 \pm 12.1$ & $101.4 \pm 12.8$ & $100.1 \pm 16.6$ & $101.2 \pm 17.0$ \\
\hline 1-hr plasma glucose (mg/dl) & $200.3 \pm 49.7$ & $207.3 \pm 51.9$ & $193.6 \pm 59.0$ & $200.8 \pm 59.2$ & $194.8 \pm 47.9$ & $181.2 \pm 48.4$ \\
\hline 2-hr plasma glucose (mg/dl) & $155.6 \pm 44.8$ & $163.8 \pm 54.0$ & $146.2 \pm 40.8$ & $164.3 \pm 54.1$ & $138.0 \pm 61.1^{*}$ & $147.6 \pm 60.8$ \\
\hline Fasting serum insulin $(\mathrm{mU} / \mathrm{ml})$ & $5.3 \pm 2.9$ & $5.8 \pm 3.7$ & $5.1 \pm 3.1$ & $7.5 \pm 3.6$ & $8.1 \pm 4.0$ & $6.8 \pm 3.2$ \\
\hline 1-hr serum insulin (mU/ml) & $47.3 \pm 33.4$ & $46.7 \pm 33.0$ & $40.9 \pm 37.5$ & $37.6 \pm 25.9$ & $46.7 \pm 37.9$ & $49.0 \pm 35.1$ \\
\hline 2-hr serum insulin $(\mathrm{mU} / \mathrm{ml})$ & $36.5 \pm 36.8$ & $41.0 \pm 26.5$ & $35.4 \pm 21.4$ & $35.8 \pm 21.5$ & $38.2 \pm 30.3$ & $46.9 \pm 31.4$ \\
\hline HbA1c (\%) & $5.28 \pm 0.6$ & $5.27 \pm 0.60$ & $5.46 \pm 0.55^{*}$ & $5.41 \pm 0.5$ & $5.46 \pm 0.60$ & $5.60 \pm 0.72$ \\
\hline HOMA-IR & $1.36 \pm 0.72$ & $1.49 \pm 0.94$ & $1.34 \pm 0.88$ & $1.84 \pm 0.88$ & $1.99 \pm 1.05$ & $1.70 \pm 0.75$ \\
\hline Systolic blood pressure (mmHg) & $138.2 \pm 13.9$ & $135 \pm 10.0$ & $128.8 \pm 21.1$ & $132.5 \pm 16.5$ & $126.8 \pm 15.1$ & $126.0 \pm 13.4$ \\
\hline Diastolic blood pressure (mmHg) & $85.0 \pm 8.8$ & $79.6 \pm 8.4^{*}$ & $66.8 \pm 13.5^{* *}$ & $79.7 \pm 12.9$ & $76.8 \pm 10.2$ & $78.6 \pm 9.3$ \\
\hline Total cholesterol (mg/dl) & $209.6 \pm 44.0$ & $216.5 \pm 25.0$ & $209.9 \pm 56.4$ & $195.1 \pm 35.2$ & $204.3 \pm 30.5$ & $193.7 \pm 28.6$ \\
\hline Triglyceride (mg/dl) & $110.0 \pm 82.7$ & $135.9 \pm 74.2$ & $92.0 \pm 40.5$ & $128.2 \pm 80.4$ & $136.6 \pm 81.8$ & $167.8 \pm 59.5$ \\
\hline HDL cholesterol (mg/dl) & $58.0 \pm 18.8$ & $55.2 \pm 9.8$ & $59.1 \pm 16.4$ & $57.8 \pm 12.2$ & $57.5 \pm 14.6$ & $56.1 \pm 12.6$ \\
\hline Free fatty acid (mEq/l) & $0.750 \pm 0.346$ & $0.812 \pm 0.380$ & $0.694 \pm 0.114$ & $0.911 \pm 0.180$ & $0.679 \pm 0.125^{* *}$ & $0.836 \pm 0.246^{*}$ \\
\hline RLP cholesterol (mg/dl) & $5.80 \pm 5.46$ & $4.79 \pm 1.62$ & $4.56 \pm 1.98$ & $5.71 \pm 2.78$ & $8.05 \pm 12.66$ & $8.35 \pm 14.35$ \\
\hline
\end{tabular}

Values are the mean \pm S.D. The significance of the change in the values in each study period (baseline vs crossover [12 week] [Period 1]; crossover vs study end point [24 week] [Period 2]) in each study group were examined by student's paired $t$-test.

$p<0.05$ and $<0.01$ are indicated by ${ }^{*}$ and ${ }^{* *}$, respectively. 
TABle 4. Percent change in the characteristic values in period 1.

\begin{tabular}{lcccc}
\hline \multicolumn{1}{c}{ Characteristics } & Control & Intervention & $p$ (t-test) & $p(\mathrm{M}-\mathrm{W})$ \\
\hline Hip circumference $(\mathrm{cm})$ & $2.3 \pm 2.5$ & $-0.2 \pm 2.4$ & $0.025^{*}$ & $0.030^{*}$ \\
Abdominal fat area : Total $\left(\mathrm{cm}^{2}\right)$ & $3.7 \pm 17.8$ & $-7.7 \pm 15.9$ & 0.119 & 0.154 \\
Visceral $\left(\mathrm{cm}^{2}\right)$ & $-5.1 \pm 23.3$ & $-17.1 \pm 26.7$ & 0.273 & 0.420 \\
2-hr plasma glucose $(\mathrm{mg} / \mathrm{dl})$ & $0.8 \pm 31.6$ & $-15.7 \pm 20.1$ & $0.038^{*}$ & 0.077 \\
Diastolic blood pressure $(\mathrm{mmHg})$ & $-6.1 \pm 7.6$ & $-2.5 \pm 12.7$ & 0.439 & 0.321 \\
Free fatty acid $(\mathrm{mEq} / \mathrm{l})$ & $18.7 \pm 52.1$ & $-22.3 \pm 21.5$ & $0.017^{*}$ & $0.030^{*}$ \\
\hline
\end{tabular}

Values are mean \pm S.D. $P<0.05$ is indicated by ${ }^{*}$. The significance of the difference between the values was examined by Student's paired $t$-test as well as Mann Whitney's U-test.

of these differences by comparing the change in their values from the baseline to the end of period 1 in the I/C group with those in the C/I group (Table 4). The percent changes from the baseline values for hip circumference, 2-hr PG levels and serum FFA levels were significantly different between the groups, while those for VFA and diastolic BP were not significantly different. The degree of change was very subtle for the hip circumference (intervention vs control: $0.2 \%$ reduction vs $2.3 \%$ increase), indicating that the change may not be clinically relevant. However, the change was substantial for both 2-hr PG levels ( $15.7 \%$ reduction vs $0.8 \%$ increase) and serum FFA levels (22.3\% reduction vs $18.7 \%$ increase). No other clinical characteristics showed significant change difference between the groups in period 1 .

\section{Crossover comparison of periods 1 and 2}

The effects of the intervention were also evaluated by comparing the percent changes in

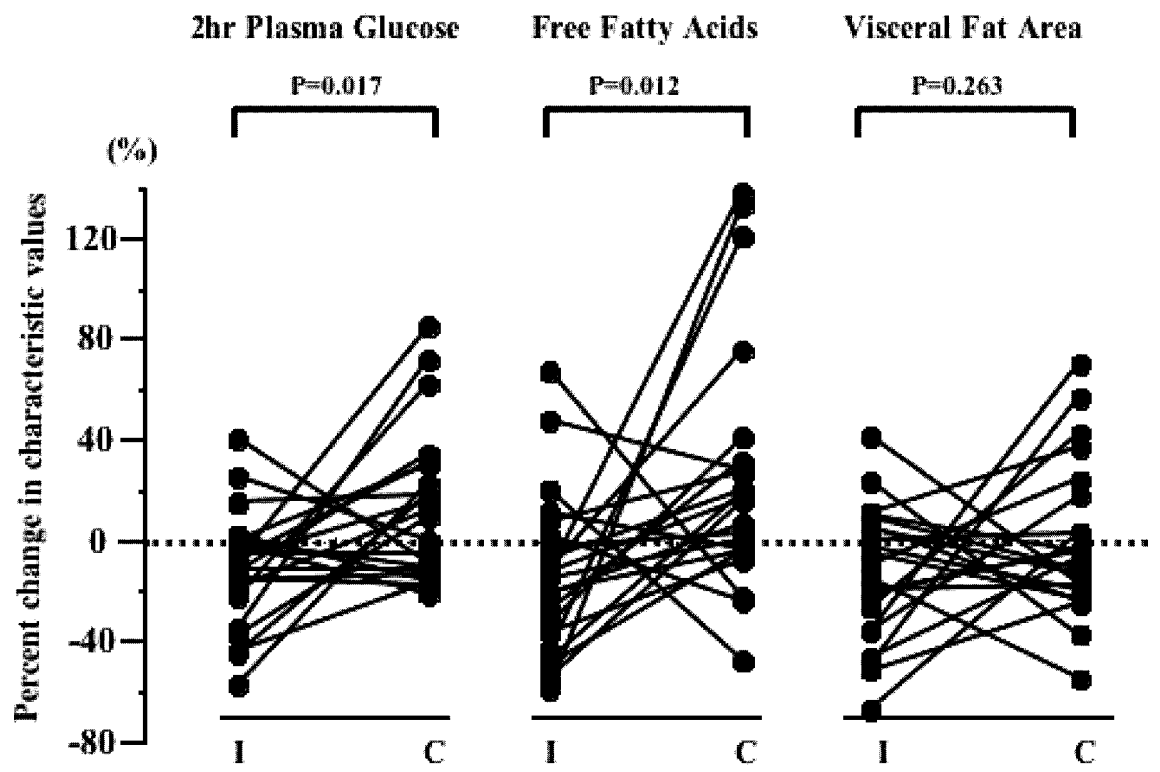

Fig. 1. Percent change in characteristic values by the intervention. Twenty-three subjects with impaired glucose tolerance were randomized in a crossover design to consume at breakfast either PBF (250 $\mathrm{kcal})$ together with foods that were $250 \mathrm{kcal}$ less than their usual breakfast (Intervention) or their usual breakfast (Control) for two 12-week periods. The changes in 2-hr plasma glucose, serum free fatty acids levels and abdominal visceral fat area in the intervention period (I) were compared with those in the control period $(\mathrm{C})$. Lines link each subject in the two treatment periods. 
the characteristic values between periods 1 and 2, or namely, between the intervention and the control periods in the same subjects (Fig. 1). Significant differences in the mean percent change were observed for 2-hr PG levels (intervention vs control: $-11.8 \pm 22.5$ vs $11.2 \pm 30.2 \%, p=0.024$ ) and serum FFA levels $(-13.6 \pm 30.0$ vs $23.1 \pm$ $46.5 \%, p=0.008)$. The percent changes in VFA $(-13.9 \pm 26.2$ vs $0.6 \pm 30.2 \%, p=0.263)$ were not significantly different between the intervention and control periods. However, as seen in Fig. 1, the subjects seemed to be divided into two groups (responders and non-responders) based on the differences in the changes in VFA between the periods. Subjects whose VFA decreased in the intervention period and, decreased less, or even increased in the control period, were defined as responders, while the others were considered as non-responders. In the responders (12 out of 22 subjects), characteristics relating to visceral obesity such as VFA $\left(86.0 \pm 35.1\right.$ vs $50.0 \pm 28.1 \mathrm{~cm}^{2}$, $p=0.017)$ and the ratio between visceral and subcutaneous fat areas (V/S ratio) $(0.95 \pm 0.43$ vs 0.38 $\pm 0.24, p=0.002$ ) were significantly higher than those in the non-responders, although BMI was not significantly different between them $(24.8 \pm 2.8$ vs $\left.24.3 \pm 3.2 \mathrm{~kg} / \mathrm{m}^{2}, p=0.724\right)$.

\section{Discussion}

We first evaluated the changes in the characteristic values from the baseline in the intervention period (period 1) in the I/C group as the effects of the intervention, and found significant decreases in VFA, 2-hr PG levels and serum FFA levels. Therefore, these decreases can be explained as beneficial effects of the intervention. However, since we have not monitored their diet record and lifestyle characteristics during the study periods, the changes in the characteristic values observed in the analysis might have occurred due to the subjects changing their lifestyle to achieve a better outcome, despite being advised not to make changes. It is also possible that these changes might be merely seasonal changes. In consideration of these possibilities, we further evaluated the differences in the change in the characteristic values between the I/C and the $\mathrm{C} / \mathrm{I}$ groups in period 1 (namely, intervention vs control in the different subjects) as well as between periods 1 and 2 in the $\mathrm{I} / \mathrm{C}$ and $\mathrm{C} / \mathrm{I}$ groups (namely, intervention vs control in the same subjects). In the I/C group, the effect and withdrawal effect of the intervention can be examined in each study period; therefore, comparing the changes in the characteristic values in period 1 with those in period 2 in the I/C group might show a more distinct difference. The decreases in 2-hr PG levels and serum FFA levels in the intervention were again significant in the above analyses, indicating that, at least, these are effects of PBF.

Very recently, acute effects of PBF ingestion on postprandial glucose levels and FFA were reported (Arai et al. 2007). In the report, consumption of PBF at breakfast has been shown to improve postprandial glucose levels after lunch, where PBF were not consumed. This effect was considered as the second-meal effect, which has been reported for consumption of a low-glycemicindex diet (Jenkins et al. 1982; Liljeberg et al. 1999). Therefore, since the effects of consumption of PBF at breakfast does not seem to be limited to breakfast only rather extended to lunch, the effects of long-term consumption of PBF may accumulate and, become more prolonged and stronger than one-time consumption. This idea further supports the decreases in 2-hr PG levels and serum FFA levels observed in the intervention to be the effects of PBF.

The decrease in VFA was significant only in analysis comparing the value at the end of the intervention period with that at the baseline in the I/C group. This may discount the probable decrease in VFA in the intervention. Analysis comparing the change in VFA in the I/C group with that in the C/I group in period 1 showed no significant difference, probably because the VFA in the $\mathrm{C} / \mathrm{I}$ group also decreased in period 1. This indicates that the decrease in VFA observed in the $\mathrm{I} / \mathrm{C}$ group in period 1 may have been due to a seasonal change. Analysis comparing the change in VFA in the intervention period with that in the control period in the same subjects also showed no significance. However, we further checked the data individually, as shown in Fig. 1. 
Interestingly, the responders were more viscerally obese than the non-responders, which can explain the reduction in VFA with the intervention being found only in the viscerally obese subjects, thus indicating that the consumption of PBF is not effective to decrease VFA in general, but is effective in subjects who are viscerally obese. Although this finding seems to limit the effect of the intervention on the reduction in VFA to viscerally obese subjects, these subjects particularly need to reduce VFA. Therefore, this result does not seem to discount the beneficial effect of the intervention, although studies with more subjects are needed to determine the detailed characteristics of subjects for whom the intervention is effective.

$\mathrm{PBF}$ is a novel palatinose-based enteral formula. Its bolus intragastric ingestion in rats was shown to suppress postprandial PG levels, and its long-term administration in rats was shown to reduce visceral fat accumulation and improve insulin sensitivity (Arai et al. 2004). In this human study, we examined the effects of PBF using the hypothesis below: postprandial glucose levels are expected to decrease whenever PBF is consumed, and thus its long-term consumption seems to induce chronically decreased postprandial PG levels, leading to improved insulin sensitivity and thus an improvement of several characteristics related to insulin resistance such as impairments of glucose tolerance and early insulin secretion in response to an oral glucose load, an accumulation of visceral fat and a dyslipidemic profile. We showed that the long-term consumption of PBF decreased 2-hr PG levels after OGTT, serum FFA levels and VFA. These findings seem to agree well with the above hypothesis; however, some results were not in good agreement with the hypothesis or our previous study with rats. The administration of PBF for 2 months in rats did not decrease serum FFA levels but serum triglyceride levels (Arai et al. 2004). The difference in species may account for this difference in the effect of PBF on the lipid profile. No improvement in early insulin secretion in response to OGTT with the intervention was significantly observed in this study. To resolve these issues, further studies with more subjects and a longer study period are thus required in the future.

In conclusion, the consumption of PBF suppressed postprandial hyperglycemia indicated as 2-hr PG levels, improved the lipid profile such as serum FFA levels and, at least in a viscerally obese population, reduced visceral fat accumulation indicated as VFA in humans.

\section{References}

Arai, H., Mizuno, A., Matsuo, K., Fukaya, M., Sasaki, H., Arima, H., Matsuura, M., Taketani, Y., Doi, T. \& Takeda, E. (2004) Effect of a novel palatinose-based liquid balanced formula (MHN-01) on glucose and lipid metabolism in male Sprague-Dawley rats after short- and long-term ingestion. Metabolism, 53, 977-983.

Arai, H., Mizuno, A., Sakuma, M., Fukaya, M., Matsuo, K., Muto, K., Sasaki, H., Matsuura, M., Okumura, H., Yamamoto, H., Taketani, Y., Doi, T. \& Takeda, E. (2007) Effects of a palatinose-based liquid diet (Inslow) on glycemic control and the second-meal effect in healthy men. Metabolism, 56, 115-121.

Bretzel, R.G., Voigt, K. \& Schatz, H. (1998) The United Kingdom Prospective Diabetes Study (UKPDS) implications for the pharmacotherapy of type 2 diabetes mellitus. Exp. Clin. Endocrinol. Diabetes, 106, 369-372.

Bruce, D.G., Chisholm, D.J., Storlien, L.H. \& Kraegen, E.W. (1988) Physiological importance of deficiency in early prandial insulin secretion in non-insulin-dependent diabetes. Diabetes, 37, 736-744.

Chiasson, J.L., Josse, R.G., Gomis, R., Hanefeld, M., Karasik, A., Laakso, M. \& STOP-NIDDM Trail Research Group (2002) Acarbose for prevention of type 2 diabetes mellitus: the STOP-NIDDM randomised trial. Lancet, 259, 2072-2077.

Dahlquist, A., Auricchio, S., Semenza, G. \& Prader, A. (1963) Human intestinal disaccharidases and hereditary disaccharide intolerance: The hydrolysis of sucrose, isomaltose, palatinose (isomaltulose), and 1,6-a-oligosaccharide (isomalto-oligosaccharide) preparation. J. Clin. Invest., 42, 556-562.

Eriksson, K.F. \& Lindgarde, F. (1998) No excess 12-year mortality in men with impaired glucose tolerance who participated in the Malmo Preventive Trial with diet and exercise. Diabetologia, 41, 1010-1016.

Hanefeld, M., Cagatay, M., Petrowitsch, T., Neuser, D., Petzinna, D. \& Rupp, M. (2004) Acarbose reduces the risk for myocardial infarction in type 2 diabetic patients: metaanalysis of seven long-term studies. Eur. Heart J., 25, 10-16.

Hernan, W.H., Brandle, M., Zhang, P., Williamson, D.F., Matulik, M.J., Ratner, R.E., Lachin, J.M. \& Engelgau, M.M. (2003) Diabetes Prevention Program Research Group. Costs associated with the primary prevention of type 2 diabetes mellitus in the diabetes prevention program. Diabetes Care, 26, 36-47.

Jebb, S.A., Cole, T.J., Doman, D., Murgatroyd, P.R. \& Prentice, A.M. (2000) Evaluation of the novel Tanita body-fat analyser to measure body composition by comparison with a four-compartment model. Br. J. Nutr., 83, 115-122. 
Jenkins, D.J., Wolever, T.M., Taylor, R.H., Griffiths, C., Krzeminska, K., Lawrie, J.A., Bennett, C.M., Goff, D.V., Sarson, D.L. \& Bloom, S.R. (1982) Slow release dietary carbohydrate improves second meal tolerance. Am. J. Clin. Nutr., 35, 1339-46.

Laakso, M. \& Lehto, S. (1997) Epidemiology of macrovascular disease in diabetes. J. Diabetes Rev., 5, 294-315.

Laedtke, T., Kjems, L., Porksen, N., Schmitz, O., Veldhuis, J., Kao, P.C. \& Butler, P.C. (2000) Overnight inhibition of insulin secretion restores pulsatility and proinsulin/insulin ratio in type 2 diabetes. Am. J. Physiol. Endocrinol. Metab., 279, E520-E528.

Liljeberg, H.G., Akerberg, A.K. \& Bjorck, I.M. (1999) Effect of the glycemic index and content of indigestible carbohydrates of cereal-based breakfast meals on glucose tolerance at lunch in healthy subjects. Am. J. Clin. Nutr., 69, 647-655.

Lillioja, S., Mott, D.M., Howard, B.V., Bennett, P.H., Yki-Jarvinen, H., Freymond, D., Nyomba, B.L., Zurlo, F., Swinburn, B. \& Bogardus, C. (1988) Impaired glucose tolerance as a disorder of insulin action. Longitudinal and cross-sectional studies in Pima Indians. N. Engl. J. Med., 318, $1217-1225$.

Lina, B.A., Jonker, D. \& Kozianowski, G. (2002) Isomaltulose (Palatinose): a review of biological and toxicological studies. Food Chem. Toxicol., 40, 1375-1381.

Lingström, P., Lundgren, F., Birkhed, D., Takazoe, I. \& Frostell, G. (1997) Effects of frequent mouthrinses with palatinose and xylitol on dental plaque. Eur. J. Oral. Sci., 102, 162-169.

Nakagami, T. \& the DECODA Study Group (2004) Hyperglycaemia and mortality from all causes and from cardiovascular disease in five populations of Asian origin. Diabetologia, 47, 385-394.

Oldroyd, J.C., Unwin, N.C., White, M., Imrie, K., Mathers, J.C. \& Alberti, K.G. (2001) Randomised controlled trial evaluating the effectiveness of behavioural interventions to modify cardiovascular risk factors in men and women with impaired glucose tolerance: outcomes at 6 months. Diabetes Res. Clin. Pract., 52, 29-43.

Reaven, G. \& Miller, R. (1968) Study of the relationship between glucose and insulin responses to an oral glucose load in man. Diabetes, 17, 560-569.
Ribeiro-Filho, F.F., Faria, A.N., Kohlmann, N.E., Zanella, M.T. \& Ferreira, S.R. (2003) Two-hour insulin determination improves the ability of abdominal fat measurement to identify risk for the metabolic syndrome. Diabetes Care, 26, 1725-1730.

Risso, A., Mercuri, F., Quagliaro, L., Damante, G. \& Ceriello, A. (2001) Intermittent high glucose enhances apoptosis in human umbilical vein endothelial cells in culture. Am. J. Physiol. Endocrinol. Metab., 281, E924-E930.

Savage, P.J., Bennion, L.J., Flock, E.V., Nagulesparan, M., Mott, D., Roth, J., Unger, R.H. \& Bennett, P.H. (1979) Dietinduced improvement of abnormalities in insulin and glucagon secretion and in insulin receptor binding in diabetes mellitus. J. Clin. Endocrinol. Metab., 48, 999-1007.

Siddiqui, I.R. \& Furgala, B. (1967) Isolation and characterization of oligosaccharides from honey. Part 1. Disaccharides. J. Api. Res., 6, 139-145.

Temelkova-Kurktschiev, T.S., Koehler, C., Henkel, E., Leonhardt, W., Fuecker, K. \& Hanefeld, M. (2000) Postchallenge plasma glucose and glycemic spikes are more strongly associated with atherosclerosis than fasting glucose or HbA1c level. Diabetes Care, 23, 1830-1834.

Tominaga, M., Eguchi, H., Manaka, H., Igarashi, K., Kato, T. \& Sekikawa, A. (1999) Impaired glucose tolerance is a risk factor for cardiovascular disease, but not impaired fasting glucose. The Funagata Diabetes Study. Diabetes Care, 22, 920-924.

UK Prospective Diabetes Study (UKPDS) Group (1998) Intensive blood-glucose control with sulphonylureas or insulin compared with conventional treatment and risk of complications in patients with type 2 diabetes (UKPDS 33). Lancet, 352, 837-853.

Uusitupa, M., Lindi, V., Louheranta, A., Salopuro, T., Lindstrom, J. \& Tuomilehto, J. (2003) Finnish Diabetes Prevention Study Group. Long-term improvement in insulin sensitivity by changing lifestyles of people with impaired glucose tolerance: 4-year results from the Finnish Diabetes Prevention Study. Diabetes, 52, 2532-2538.

World Health Organization (1999) Definition, diagnosis and classification of diabetes mellitus and its complications. Geneva, World Health Organization. (Report of a WHO consultation. Part 1, diagnosis and classification of diabetes Mellitus) 